\title{
光镊技术研究硝酸铵在超粘气溶胶中的挥发性
}

\author{
吕席卷 ${ }^{a} \quad$ 张暳宏*, \\ $\left({ }^{a}\right.$ 北京理工大学材料学院 北京 100081$)$ \\ ( ${ }^{b}$ 北京理工大学化学与化工学院 北京 100081)
}

\begin{abstract}
摘要 大气颗粒物中挥发性物质的气粒分配问题是大气科学研究的热点. 选择典型的高粘度态模型体系、硝酸铵/蔗糖 体系以及硝酸铵/硫酸镁体系, 利用光镊一受激拉曼光谱技术原位获得液滴的自发拉曼和受激拉曼信号, 同时观察回音 壁(WGM)模式, 利用米氏散射理论对一系列的 WGMs 峰位在给定范围内的粒子半径和折射率进行模拟计算, 通过 Maxwell 方程精确计算了两个体系中硝酸铵在不同相对湿度 $(\mathrm{RH})$ 下的有效饱和蒸汽压值, 结果表明, 在低湿度下的超 粘态液滴中硝酸铵的有效饱和蒸汽压比纯硝酸铵的饱和蒸汽压低 $1 \sim 3$ 个数量级. 显然, 低相对湿度下, 液滴中硝酸铵 的挥发受到了抑制.

关键词 光铌; 硝酸铵; 挥发性; 蒸汽压; 超粘态
\end{abstract}

\section{Volatility of Ammonium Nitrate in Ultra-viscous Aerosol Droplets by Optical Tweezers}

\author{
Lü, Xijuan ${ }^{a} \quad$ Zhang, Yunhong ${ }^{*, b}$ \\ $\left({ }^{a}\right.$ School of Materials Science \& Engineering, Beijing Institute of Technology, Beijing 100081) \\ ( ${ }^{b}$ School of Chemistry and Chemical Engineering, Beijing Institute of Technology, Beijing 100081)
}

\begin{abstract}
The partitioning of volatile substances in atmospheric particulates is a hot topic in atmospheric science. Ammonium nitrate $\left(\mathrm{NH}_{4} \mathrm{NO}_{3}\right)$ is an important inorganic component in atmospheric aerosols, which is a salt with relatively high vapor pressure. Particles containing $\mathrm{NH}_{4} \mathrm{NO}_{3}$ are equilibrium with gaseous $\mathrm{NH}_{3}$ and $\mathrm{HNO}_{3}$ and the partitioning between particle and gas is a strong function of temperature and relative humidity. Atmospheric aerosols have both natural and anthropogenic sources and consist of both organic and inorganic components, and many of them will likely form in semisolid, glassy, and high viscous state in the atmosphere, which show nonequilibrium kinetic characteristics at low relative humidity conditions. In this research, in order to understand the volatility of ammonium nitrate in ultra-viscous aerosol droplets, optical tweezers coupled with cavity-enhanced Raman spectroscopy were employed to observe the volatility of ammonium nitrate in the mixture of $\mathrm{NH}_{4} \mathrm{NO}_{3} / \mathrm{MgSO}_{4}$ and $\mathrm{NH}_{4} \mathrm{NO}_{3}$ /sucrose droplets. Optical tweezers-stimulated Raman spectroscopy, compared with other suspension techniques, can not only suspend droplets, but also obtain the chemical composition, structure of droplets and other information according to the conventional Raman scattering spectra of droplets. The radius and refractive index of droplets can be calculated according to stimulated Raman-Mie scattering resonance. The advantages of optical tweezers stimulated Raman spectroscopy are that particle radius can be accurately measured, chemical composition, phase and shape can be controlled, and long-term observation can be realized. Here, the radii and refractive indexes of the levitated droplets were determined in real time using the wavelength positions of stimulated Raman spectra, and the effective vapor pressures of ammonium nitrate at different relative humidity $(\mathrm{RH})$ were obtained according to Maxwell equation. For the droplets with ammonium nitrate/sucrose molar ratio of $1: 1$, the effective vapor pressure of ammonium nitrate decreased with the decrease of RH. When the RH decreased from $70 \%$ to $20 \%$, the effective vapor pressure of ammonium nitrate decreased from (3.577士 $0.82) \times 10^{-5}$ to $(6.55 \pm 1.36) \times 10^{-6} \mathrm{~Pa}$, compared to the vapor pressure of pure ammonium nitrate $(1.67 \pm 0.24) \times 10^{-3}$ to $(6.64 \pm 0.3) \times 10^{-3} \mathrm{~Pa}$, the evaporation of ammonium nitrate in the mixed droplet was suppressed by sucrose, especially at low RH. For the mixed droplets with ammonium nitrate/magnesium sulfate molar ratio of $1: 1$, a similar phenomenon was observed. When the RH decreased from $70 \%$ to $40 \%$, the effective vapor pressure of ammonium nitrate decreased from $(4.38$ $\pm 0.21) \times 10^{-3}$ to $(8.13 \pm 2.34) \times 10^{-5} \mathrm{~Pa}$. The results showed that the effective saturated vapor pressures of ammonium nitrate in ultra-viscous droplets at low humidity were $1 \sim 3$ orders lower than those of pure ammonium nitrate. Obviously, the volatilization of ammonium nitrate in droplets was inhibited at low relative humidity.

Keywords optical tweezers; ammonium nitrate; volatility; vapor pressure; ultra-viscous
\end{abstract}

\footnotetext{
* E-mail: yhz@bit.edu.cn

Received October 15, 2019; published March 11, 2020.

Project supported by the National Natural Science Foundation of China (No. 91544223).

项目受国家自然科学基金(No. 91544223)资助.
} 


\section{1 引言}

硝酸铵 $\left(\mathrm{NH}_{4} \mathrm{NO}_{3}\right)$ 和硫酸铵 $\left[\left(\mathrm{NH}_{4}\right)_{2} \mathrm{SO}_{4}\right]$ 是城市细颗 粒物的重要成分. 近年来, 我国大气颗粒物中硫酸盐大 幅减少, 硝酸盐占比持续增加 ${ }^{[1,2]}$. $\mathrm{NH}_{4} \mathrm{NO}_{3}$ 由于具有较 强的吸湿性, 对大气气溶胶的成云能力以及全球辐射平 衡有着非常重要的影响. 同其它无机气溶胶不同的是, $\mathrm{NH}_{4} \mathrm{NO}_{3}$ 气溶胶具有相对较高的蒸汽压. 受湿度和温度 的影响, $\mathrm{NH}_{4} \mathrm{NO}_{3}$ 颗粒和 $\mathrm{NH}_{3}(\mathrm{~g})+\mathrm{HNO}_{3}(\mathrm{~g})$ 之间存在着 气固分配平衡. 因此, 研究 $\mathrm{NH}_{4} \mathrm{NO}_{3}$ 蒸汽压值与化学组 成、相态、温度以及相对湿度的依赖关系, 对于认清雾 皬的形成机制具有重要的科学意义.

大气气溶胶包括有机气溶胶和无机气溶胶. 硫酸镁 $\left(\mathrm{MgSO}_{4}\right)$ 气溶胶在低相对湿度下呈胶态, 这种胶态结构 会阻碍水分子在液滴内部的传递 ${ }^{[3]}$. 有机气溶胶可以在 大气中形成半固态、玻璃态和高粘性状态, 气溶胶的高 粘性状态会抑制气溶胶体相内的传质, 减缓水的吸收或 蒸发速度. 蔗糖气溶胶在低相对湿度下能形成玻璃态, 是典型的高粘态气溶胶. 粘性气溶胶中的水、有机物和 氧化剂等的缓慢输送可导致气溶胶存在瞬态状态, 这些 状态不完全受热力学原理支配, 而是由气粒分配的动力 学支配 ${ }^{[4]}$.

不同组分的挥发性和半挥发性气溶胶的生成、转 化、气粒分配平衡、气粒化学反应一向是大气气溶胶研 究领域的关注重点 ${ }^{[5,6]}$. 已有不少研究者通过不同技术 手段讨论了 $\mathrm{NH}_{4} \mathrm{NO}_{3}$ 的挥发性 ${ }^{[7 \sim 9]}$, 我们之前的工作中, 也通过光镊受激拉曼技术研究了 $\mathrm{NH}_{4} \mathrm{NO}_{3}$ 的挥发, 计算 了 $\mathrm{NH}_{4} \mathrm{NO}_{3}$ 的饱和蒸汽压以及挥发通量 ${ }^{[10]}$. 但是, 关于 硝酸铵在超粘态气溶胶中的挥发性的数据至今还没有. 选择典型的超粘态体系 $\mathrm{NH}_{4} \mathrm{NO}_{3} / \mathrm{MgSO}_{4}$, 以及 $\mathrm{NH}_{4} \mathrm{NO}_{3} /$ 蔗糖体系, 利用光铌受激拉曼光谱技术研究了硝酸铵在 超粘气溶胶中的挥发性. 利用稳态传质模型 Maxwell 公 式分别计算了两个体系中硝酸铵在不同相对湿度下的 有效饱和蒸汽压值.

\section{2 结果与讨论}

图 1(a)展示了光铝捕获的摩尔比 $1: 1$ 的硝酸铵/蔗 糖液滴在相对湿度 $73 \%$ 以及 $63 \%$ 时的 WGM 共振模式的 波长随时间推移的变化情况, 利用米氏散射理论对一系 列的 WGMs 峰位在给定范围内的粒子半径和折射率进 行模拟计算, 计算结果如图 1(b)所示, 可以看到液滴半 径和折射率随 $\mathrm{RH}$ 的变化情况.

硝酸铵液滴的摩尔分数 $\chi_{i}$, 活度系数 $f_{i}$ 与 $\mathrm{RH}$ 的关 系通过 AIOMFAC 热力学模型模拟得到如图 2 所示.

根据实验结果利用麦克斯韦方程计算的不同相对 湿度下硝酸铵在粘态物质熫糖和硫酸镁中的有效饱和 蒸汽压值如图 3 所示. 从图中可以直观看出纯硝酸铵的 饱和蒸汽压以及硝酸铵/蔗糖、硝酸铵/硫酸镁中的硝 (a)

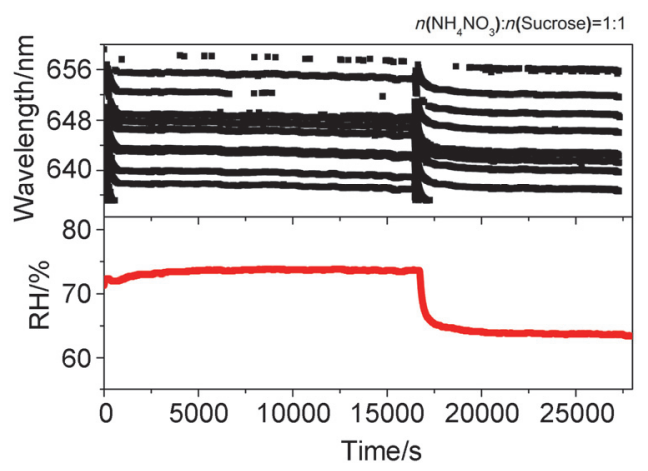

(b)

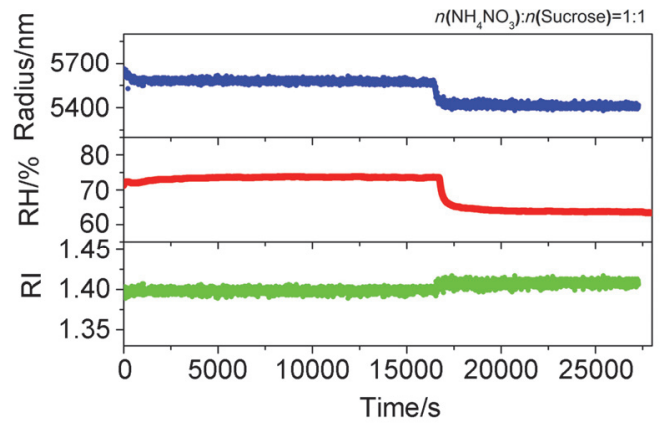

图 1 (a) $\mathrm{NH}_{4} \mathrm{NO}_{3}$ / 蔗糖液滴在 $\mathrm{RH}$ (红线)分别为 $73 \%$ 和 $63 \%$ 时 $\mathrm{WGM}$ 共振模式的波长(黑线)随时间推移的变化情况; (b) $298 \mathrm{~K}$ 时, 光铌中 悬浮的 $\mathrm{NH}_{4} \mathrm{NO}_{3}$ /蔗糖液滴在不同湿度(红线)下液滴半径(蓝线)和折射 率(绿线)随时间的变化

Figure 1 (a) Time evolution of the wavelengths of the WGM resonant modes on the $\mathrm{OH}$ band for the $\mathrm{NH}_{4} \mathrm{NO}_{3}$ /sucrose at $73 \%$ and $63 \% \mathrm{RH}$; (b) Time-dependent $\mathrm{NH}_{4} \mathrm{NO}_{3}$ /sucrose droplet size (blue line), RI (green line) and RH (red line) at $298 \mathrm{~K}$ in the optical tweezers

酸铵在不同相对湿度下有效饱和蒸汽压的分布范围. 研 究表明蔗糖和硫酸镁在相对湿度降低的过程中粘度会 逐渐增大，当相对湿度低至 $23 \%$ 左右蔗糖会形成玻璃 态，硫酸镁则在相对湿度低至 $40 \%$ 时成胶态 ${ }^{[3,11]}$. 对于 硝酸铵与蔗糖摩尔比为 $1: 1$ 的液滴, 硝酸铵的有效饱 和蒸气压随相对湿度的降低而降低. 当相对湿度从 $70 \%$ 下降到 $20 \%$ 时, 硝酸铵的有效饱和蒸气压从 $(3.577 \pm$ $0.82) \times 10^{-5} \mathrm{~Pa}$ 下降到 $(6.55 \pm 1.36) \times 10^{-6} \mathrm{~Pa}$ ，与纯硝酸 铵的蒸气压 $(1.67 \pm 0.24) \times 10^{-3} \mathrm{~Pa} \sim(6.64 \pm 0.3) \times 10^{-3}$ $\mathrm{Pa}$ 相比, 蔗糖对硝酸铵在混合液滴中的蒸发有抑制作 用, 尤其是在低相对湿度下. 对于硝酸铵/硫酸镁摩尔比 为 $1: 1$ 的混合液滴, 观察到类似现象. 当相对湿度从 $70 \%$ 下降到 $40 \%$ 时, 硝酸铵的有效饱和蒸气压从(4.38土 $0.21) \times 10^{-3} \mathrm{~Pa}$ 下降到 $(8.13 \pm 2.34) \times 10^{-5} \mathrm{~Pa}$. 结果表明, 在低湿度下, 超粘性液滴中硝酸铵的有效饱和蒸气压比 纯硝酸铵低 $1 \sim 3$ 个数量级. 这主要是因为低湿度下水 分的传质受阻现象阻碍了硝酸铵的挥发, 使得硝酸铵的 有效饱和蒸汽压值减小. Reid 等 ${ }^{[4]}$ 在马来酸/蔗糖体系的 挥发性研究中也发现了类似的现象, 当 RH 在 $12 \%$ 时马 来酸的有效饱和蒸汽压比较高湿度 $70 \%$ 时马来酸的有 效饱和蒸汽压低两个数量级. 这表明, 干燥过程也就是 降湿过程可以改变液滴的粘性，从而影响水分的传输动 力学特性, 进而影响挥发性物质硝酸铵的挥发. 

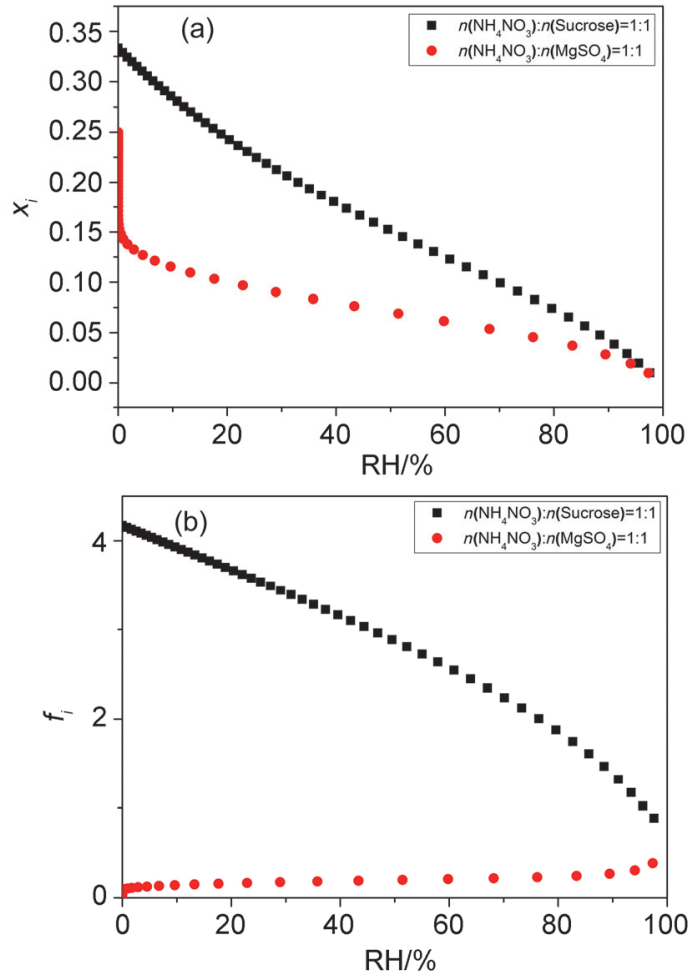

图 2 AIOMFAC 模型得到的摩尔比 $1: 1$ 的 $\mathrm{NH}_{4} \mathrm{NO}_{3}$ /蔗糖体系以及 $\mathrm{NH}_{4} \mathrm{NO}_{3} / \mathrm{MgSO}_{4}$ 两个体系中 $\mathrm{NH}_{4} \mathrm{NO}_{3}$ 液滴摩尔分数 $\chi_{i}(\mathrm{a})$, 活度系数 $f_{i}$ (b)与相对湿度的关系

Figure 2 Values of $\chi_{i}(\mathrm{a}), f_{i}$ (b) at different RHs by AIOMFAC Model for the $n_{\mathrm{NH} 4 \mathrm{NO} O}: n_{\text {sucrose }}=1: 1$ (black), $n_{\mathrm{NH} 4 \mathrm{NO} 3}: n_{\mathrm{MgSO} 4}=1: 1$ (red)

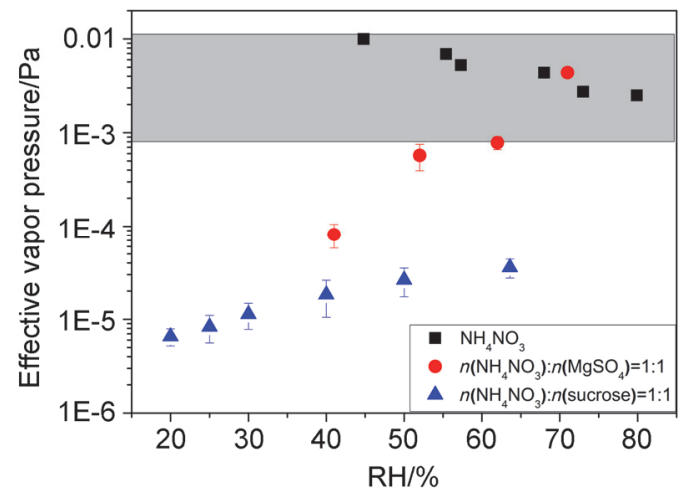

图 $3 \mathrm{NH}_{4} \mathrm{NO}_{3} /$ /葉糖, 以及 $\mathrm{NH}_{4} \mathrm{NO}_{3} /$ / 硫酸镁体系中 $\mathrm{NH}_{4} \mathrm{NO}_{3}$ 的有效饱 和蒸汽压值, 灰色区域是文献报道的不同技术手段得到的纯 $\mathrm{NH}_{4} \mathrm{NO}_{3}$ 的蒸气压值

Figure 3 Estimates of the effective vapor pressure of $\mathrm{NH}_{4} \mathrm{NO}_{3} /$ sucrose and $\mathrm{NH}_{4} \mathrm{NO}_{3} / \mathrm{MgSO}_{4}$ at each $\mathrm{RH}$ step tend toward the reported pure component vapor pressure of aqueous $\mathrm{NH}_{4} \mathrm{NO}_{3}$ (gray band) at high $\mathrm{RHs}$

\section{3 结论}

针对低湿度下玻璃态、胶态的形成过程与水分的传 质受阻现象, 选择典型的高粘度态模型体系, 硝酸铵/蔗 糖体系以及硝酸铵/硫酸镁体系, 研究硝酸铵气溶胶在 粘性物质蔗糖和硫酸镁中的挥发性, 与硝酸铵/水体系
作对比，讨论粘态物质水分传质受阻现象对半挥发性物 质硝酸铵挥发性的影响. 用液滴有效饱和蒸汽压值衡量 硝酸铵气溶胶在玻璃态、胶态物质中的挥发性. 结果表 明, 在相对湿度较低的高粘态体系中, 硝酸铵的有效饱 和蒸汽压比硝酸铵/水体系中硝酸铵的饱和蒸汽压值低 1 3 个数量级, 表明高粘态体系的传质受阻特性阻碍 了硝酸铵的挥发.

\section{4 实验与数据处理方法部分}

\section{1 制样}

将硝酸铵、蔗糖、硫酸镁分别溶解在去离子水中, 分 别配置成硝酸铵/蔗糖、硝酸铵/硫酸镁摩尔比 $1: 1$ 的硝 酸铵/蔗糖溶液, 硝酸铵/硫酸镁溶液, 通过医用/超声雾 化器(Yuyue 402AI model)雾化产生气溶胶颗粒, 气溶胶 颗粒再通过喷嘴进入样品池. 雾化器产生的气溶胶流导 入样品池后单个液滴(粒径 $1 \sim 10 \mu \mathrm{m}$ ) 能够随机地被 5 $15 \mathrm{~mW}$ 的激光束捕获. 液滴的直径可以通过继续通入 雾化的颗粒使液滴碰撞融合而增大. 样品池一共三个 口, 干湿氮气进行调节 $\mathrm{RH}$, 其中湿氮气是由干氮气通 过去离子水产生的, 用两个独立的质量流量计 (Alicat scientific)控制干氮气和湿氮气的流速流量, 干氮气和湿 氮气在进入样品池前混合均匀, 两束气流的流速控制在 $200 \mathrm{~mL} \cdot \mathrm{min}^{-1}$.

\section{2 实验装置}

光镊的激光对粒子的作用力分为两类: 散射力由光 的反射产生，梯度力由光的折射产生，当散射力和梯度 力达到平衡的时候, 液滴会被稳定的捕获. 光镊-拉曼 系统有激光产生部分、液滴捕获部分、图像收集设备、 拉曼信号收集设备组成. 激光由激光器发出先经过几个 反射镜, 然后经过透镜组, 再通过二向色镜反射进入分 束镜, 通过 $100 \times$ 浸油显微物镜聚焦形成光学陷阱, 所 用的为非荧浸油(Cargille, type FF, $n=1.48$ ), 位于 455 $\mathrm{nm}$ 蓝色 LED 灯给捕获液滴成像提供光源, 蓝光通过分 束镜和二向色镜，再通过短波通移除激光散射光，最后 投射到照相机上，通过照相机可以对捕获液滴进行平面 成像. 液滴的反向拉曼散射信号先由长波通滤去有激光 和 LED 光波长范围的光, 然后聚焦到拉曼光谱仪狭缝, 采集光谱的时间分辨为 $1 \mathrm{~s}$ (图 4).

\section{3 光谱采集}

用温湿度检测器(Center 313)来测量相应的温度和 湿度, 所测的湿度误差为 $\pm 2 \%$, 温度为 $\pm 0.5{ }^{\circ} \mathrm{C}$. 光谱 仪的型号为 Omni- 25006 , 使用光谱仪时需要冷却至 $-55{ }^{\circ} \mathrm{C}$ 来除去杂音. 一般常用的光栅是 $1200 \mathrm{~g} / \mathrm{mm}$. 实 验所用的中心波长是 $640 \mathrm{~nm}, \mathrm{WGMs}$ 的波段是 622 到 $660 \mathrm{~nm}$, 这也是水的 $\mathrm{OH}$ 伸缩振动所在的峰位置. 液滴 一旦被捕获就可以收集到自发拉曼信号和受激拉曼信 号，同时观察到回音壁(WGM)模式，每张光谱都是记录 


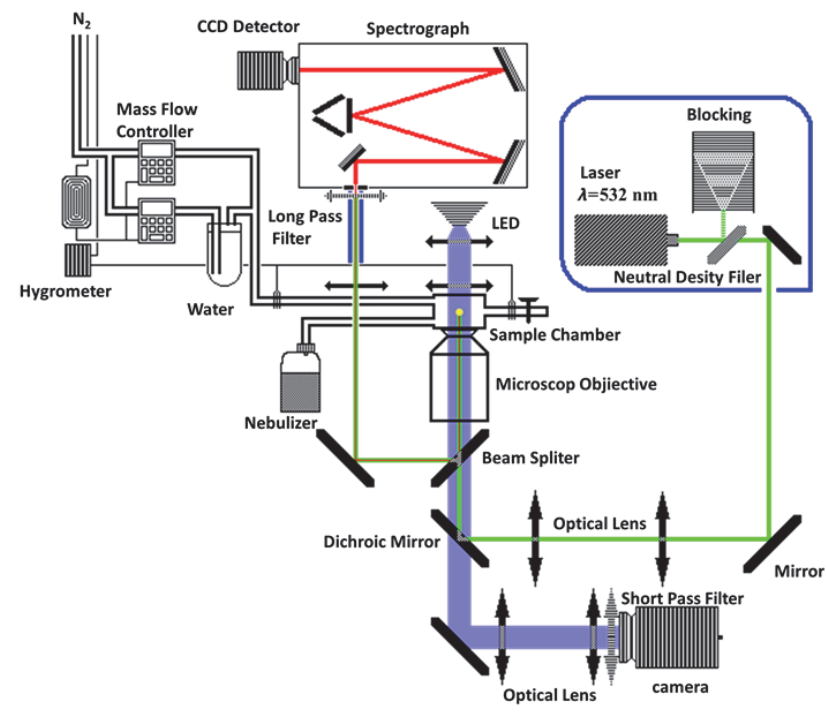

图 4 单液滴光铌实验装置示意图

Figure 4 Schematic diagram of experimental set-up used for single droplet optical tweezing

在独立的文件. 利用米氏散射理论对一系列的 WGMs 峰位在给定范围内的粒子半径和折射率进行模拟计算. 每个模拟的 WGM 都会与实验得到的比较以确定 WGM 的模级 $l$, 模数 $\mathrm{n}$ 和偏振方向 $p$, 然后计算出液滴的半径 和折射率. 因此, 通过该方法可以得到实验过程中的所 有时刻的半径和折射率信息. 这与以往的悬浮技术相 比, 激光作为光源, 一方面可以悬浮液滴, 同时该液滴 的常规拉曼散射光谱, 能提供液滴的化学组成、结构信 息, 液滴的受激拉曼散射-Mie 氏共振散射, 则可以提供 液滴的折射率、尺寸信息.

\section{4 数据处理方法}

\subsection{1 蒸汽压的计算}

由于 $\mathrm{NH}_{4} \mathrm{NO}_{3}$ 液滴和 $\mathrm{NH}_{3}(\mathrm{~g})$ 以及 $\mathrm{HNO}_{3}(\mathrm{~g})$ 之间存在 着气粒分配平衡如 Eq. 1 所示.

$$
\mathrm{NH}_{4} \mathrm{NO}_{3} \text { (aq.) } \leftrightarrows \mathrm{NH}_{3}(\mathrm{~g})+\mathrm{HNO}_{3}(\mathrm{~g})
$$

因此, 根据液滴的表面分压 $p_{r}$ 和纯物质的饱和蒸 汽压 $p^{0}$ 的关系: $p_{r}=\chi_{i} f_{i} p_{i}^{0}$, Maxwell 气相扩散定律可 变形为 Eq. $2^{[12]}$, 结合液滴半径的变化可求得体系中 $\mathrm{NH}_{4} \mathrm{NO}_{3}$ 的饱和蒸汽压 $p_{\text {tot }}$.

$$
p_{\mathrm{tot}}=p_{\mathrm{NH}_{3}}+p_{\mathrm{HNO}_{3}}=-\frac{1}{2} \frac{\mathrm{d} r^{2}}{\mathrm{~d} t} R T \frac{\rho_{\mathrm{AN}} F_{i}}{M_{\mathrm{AN}} \chi_{i} f_{i}}\left(\frac{1}{D_{\mathrm{NH}_{3}}}+\frac{1}{D_{\mathrm{HNO}_{3}}}\right)
$$

其中, $M_{\mathrm{AN}}$ 是硝酸铵的摩尔质量, $D_{\mathrm{NH} 3}$ 和 $D_{\mathrm{HNO} 3}$ 是氨气和 硝酸分别在 $\mathrm{N}_{2}$ 中的扩散系数, $R$ 是理想气体常数, $T$ 是温 度值, $f_{i}$ 代表活度系数, $\rho$ 代表液滴的密度, $F_{i}$ 是硝酸铵在 液滴中的质量分数, $\chi_{i}$ 代表硝酸铵的摩尔分数.

\subsection{2 摩尔分数 $\chi_{i}$, 活度系数 $f_{i}$ 的计算}

硝酸铵液滴的摩尔分数 $\chi_{i}$, 活度系数 $f_{i}$ 与 $\mathrm{RH}$ 的关 系通过 AIOMFAC 热力学模型模拟得到. AIOMFAC 热 力学模型是用以计算无机、有机或无机-有机宽浓度范 围内溶液组分活度系数的热力学模型, 目前已得到广泛 的应用. 模型中的有机物和无机物是大气颗粒物中的常 见物质, 通过计算活度系数可以详细阐明水溶液中无机 离子、有机官能团之间的相互作用. AIOMFAC 相对于其 它化学热力学活度系数模型, 有以下几个优势: 可以计 算从稀溶液到过饱和溶液以及结晶相中水、电解质和有 机物的活度系数, 能预测有机无机混合物的非理想性. AIOMFAC 模型适用范围较宽, 可以计算多种有机物、7 种无机阳离子 $\left(\mathrm{H}^{+} 、 \mathrm{Li}^{+} 、 \mathrm{Na}^{+} 、 \mathrm{~K}^{+} 、 \mathrm{NH}_{4}^{+} 、 \mathrm{Mg}^{2+}\right.$ 和 $\left.\mathrm{Ca}^{2+}\right)$ 和 5 种无机阴离子 $\left(\mathrm{Cl}^{-} 、 \mathrm{Br}^{-} 、 \mathrm{NO}_{3}^{-} 、 \mathrm{HSO}_{4}^{-}\right.$和 $\mathrm{SO}_{4}^{2-}$ ). 液滴的 $\rho F_{i}$ 质量浓度可以根据质量浓度和折射 率的关系结合拟和出来的实际折射率数据得出.

\section{References}

[1] Sun, J.; Liu, L.; Xu, L.; Wang, Y.; Wu, Z.; Hu, M.; Shi, Z.; Li, Y.; Zhang, X.; Chen, J.; Li, W. J. Geophys. Res. 2018, 123, 1234.

[2] Zhang, X. Y.; Wang, J. Z.; Wang, Y. Q.; Liu, H. L.; Sun, J. Y.; Zhang, Y. M. Atmos. Chem. Phys. 2015, 15, 12935.

[3] Cai, C.; Tan, S.; Chen, H.; Ma, J.; Wang, Y.; Reid, J. P.; Zhang, Y. Phys. Chem. Chem. Phys. 2015, 17, 29753.

[4] Marshall, F. H.; Miles, R. E. H.; Song, Y.-C.; Ohm, P. B.; Power, R. M.; Reid, J. P.; Dutcher, C. S. Chem. Sci. 2016, 7, 1298.

[5] Hu, M.; Shang, D.; Guo, S.; Wu, Z. Acta Chim. Sinica 2016, 74 385. (胡敏, 尚冬杰, 郭松, 吴志军, 化学学报, 2016, 74, 385.)

[6] Guo, S.; Hu, M.; Shang, D.; Guo, Q.; Hu, W. Acta Chim. Sinica 2014, 72, 145. (郭松, 胡敏, 尚冬杰, 郭庆丰, 胡伟伟, 化学学报, 2014, 72, 145.)

[7] Hu, D.; Chen, J.; Ye, X.; Li, L.; Yang, X. Atmos. Environ. 2011, 45, 2349.

[8] Chien, W.-M.; Chandra, D.; Lau, K. H.; Hildenbrand, D. L.; Helmy, A. M. J. Chem. Thermodyn. 2010, 42, 846.

[9] Hong, J.; Äijälä, M.; Häme, S. A. K.; Hao, L. Q.; Duplissy, J.; Heikkinen, L. M.; Nie, W.; Mikkilä, J.; Kulmala, M.; Prisle, N. L.; Virtanen, A.; Ehn, M.; Paasonen, P.; Worsnop, D. R.; Riipinen, I.; Petäjä, T.; Kerminen, V.-M. Atmos. Chem. Phys. 2017, 17, 4387.

[10] Lv, X.; Gao, X.; Ma, J.; Zhang, Y. Spectrosc. Spect. Anal. 2019, 39, 1648.

[11] Wang, L. N.; Cai, C.; Zhang, Y. H. J. Phys. Chem. B 2017, 121, 8551 .

[12] Krieger, A. A.; Zardini, A. A.; Krieger, U. K. Opt. Express 2009, 17, 4659 . 